\title{
The spectrum of pancreatic exocrine and endocrine (Beta-cell) function in tropical calcific pancreatitis
}

\author{
C. S. Yajnik ${ }^{1}$, K. M. Shelgikar ${ }^{1}$, R. A. Sahasrabudhe ${ }^{1}$, S. S. Naik ${ }^{1}$, V.R. Pai ${ }^{1}$, K. G. M. M. Alberti ${ }^{2}$, T. D. R. Hockaday ${ }^{3}$, \\ A. Katrak ${ }^{4}$ and P. Dandona ${ }^{4}$ \\ ${ }^{1}$ Wellcome Diabetes Study, King Edward Memorial Hospital, Pune, India, ${ }^{2}$ Department of Medicine, University of Newcastle-Upon-Tyne, \\ ${ }^{3}$ Sheikh Rashid Diabetes Unit, Radcliffe Infirmary, Oxford, and ${ }^{4}$ Metabolic Unit, Royal Free Hospital, London, U K
}

\begin{abstract}
Summary. Exocrine pancreatic marker (immunoreactivetrypsin) and endocrine Beta-cell function (plasma insulin and C-peptide during an oral glucose tolerance test) were studied in 40 subjects with tropical-calcific-pancreatitis [seven non-diabetic, seven with impaired-glucose-tolerance and 26 diabetic (fibro-calculous-pancreatic-diabetes)]. In non-diabetic and impaired-glucose-tolerance subjects there was evidence of active pancreatitis in some and exocrine function was partially preserved. Fibro-calculous-pancreatic-diabetic subjects showed severely diminished exocrine pancreatic function; none showed 'pancreatitic' elevation of immunoreactive-trypsin. Beta-cell function was preserved in non-diabetic and impaired-glucose-tolerance subjects;
\end{abstract}

diabetic subjects showed variable Beta-cell function but it was severely diminished in more than $75 \%$. Immunoreactive-trypsin and C-peptide were directly correlated $\left(r_{\mathrm{s}}=0.55\right.$, $p<0.01$ ). This cross sectional study demonstrates, for the first time, that the Beta-cell loss in tropical-calcific-pancreatitis is related to the exocrine loss. It suggests that diabetes in tropical-calcific-pancreatitis is either secondary to pancreatitis or that a common factor(s) acts simultaneously on both components.

Key words: Tropical-calcific-pancreatitis, fibro-calculouspancreatic-diabetes, immunoreactive-trypsin, C-peptide, immunoreactive insulin, oral glucose tolerance test.
Tropical calcific pancreatitis (TCP) is a form of chronic pancreatitis seen in tropical developing countries [1]. It affects young individuals, many of whom develop diabetes mellitus in the second and third decades of life [2]. The exact aetiology of TCP is unknown but 'malnutrition', and dietary toxins are thought to contribute to its development $[1,3]$. Since a World Health Organisation Study Group [4] named the diabetes secondary to TCP as fibro-calculous-pancreatic-diabetes (FCPD) and recognised it as a subclass of so called malnutrition-relateddiabetes, TCP has attracted much attention. Reports of the clinical features [1,2], descriptions of Beta-cell function in FCPD of different severity [5], and comparisons with insulin-dependent and non-insulin-dependent diabetic patients have been published [6-10]. There are, however, no reports of the interrelationship of pancreatic exocrine-endocrine function in TCP, which includes nondiabetic and impaired-glucose-tolerance subjects besides fibro-calculous-pancreatic diabetes. We report here the clinical and biochemical features, pancreatic Beta-cell markers (insulin and C-peptide) and a pancreatic exo- crine marker (immunoreactive-trypsin) in our subjects with TCP.

\section{Subjects and methods}

Subjects included in the study were from the King Edward Memorial Hospital and Sassoon General Hospital, Pune, India. These are the two largest referral centres in Pune, and serve a large urban and rural population in Pune district. The 40 TCP subjects studied represent over $80 \%$ of TCP cases diagnosed in these two hospitals during a 3 year period. Diagnosis of TCP was suspected because of a history of abdominal pain suggestive of pancreatitis. Plain $x$-ray of the abdomen showed pancreatic calculi in all subjects, confirmed by ultrasound. None of the patients presented a history of alcoholism. Ultrasound showed that no patients had obstructive hepatobiliary disease. Many $(n=20)$ had presented with symptoms of severe diabetes without ketosis or ketonuria. All subjects underwent a $75 \mathrm{~g}$ $(1.75 \mathrm{~g} / \mathrm{kg}$ in children below 15 years) oral glucose tolerance test (OGTT) on presentation. Serial blood samples were obtained via an indwelling cannula in an antecubital vein. Two baseline samples were averaged to give the fasting pre-oral glucose value. Plasma glucose (glucose oxidase), cholesterol, triglycerides and albumin were 

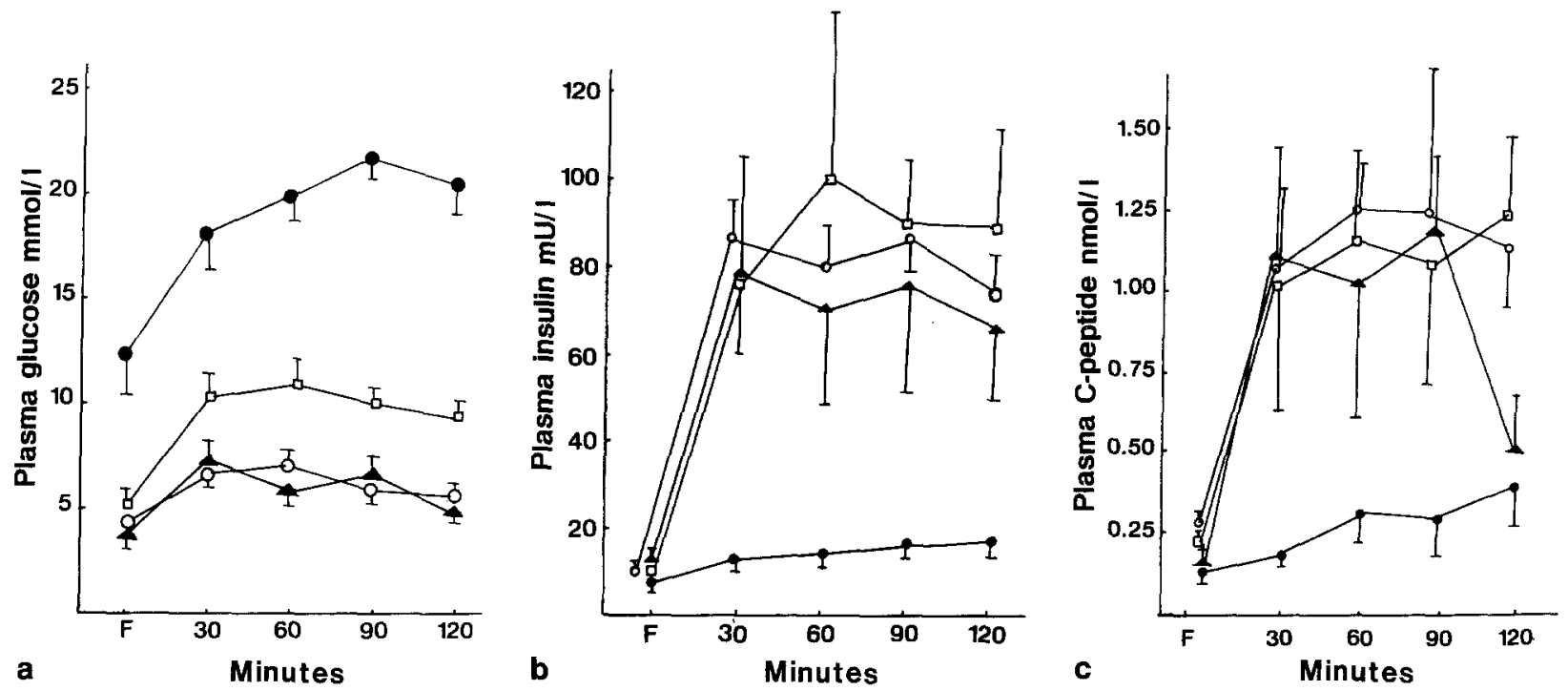

Fig. 1 a-c. Plasma glucose (a), insulin (b) and C-peptide (c) concentrations during oral glucose tolerance test. Mean \pm SEM shown. F refers to fasting. ( $\longrightarrow$ ) Non-diabetic tropical calcific pancreatitis (ND-TCP); $(\square-\square)$ TCP with impaired glucose tolerance (IGTTCP); $(\longrightarrow)$ Fibro-calculous-pancreatic-diabetes (FCPD); $(0-0)$ Control subjects (ND-C)

estimated on an Abbott VP-Super autoanalyser (Irving, Texas, USA) by using standard kits. $\mathrm{HbA}_{1}$ was measured by a 'chemical' method using fructose standards [11]. Plasma insulin was assayed (in subjects not treated with insulin) by a double antibody radioimmunoassay [12] using SAC-CEL separation (Solid Phase Second Antibody Coated Cellulose Suspension, Immunodiagnostic Systems Ltd., Tyne and Wear, U.K.), intra-batch coefficient of variation (c.v.) $<5 \%$, inter-batch $<8 \%$. Plasma C-peptide was measured using a kit (Novo, Bagsvaerd, Denmark) [13], intra-batch c.v. $<5 \%$, interbatch $<8 \%$. Immunoreactive-trypsin was assayed on fasting plasma samples by a specific radioimmunoassay (Hoechst, Frankfurt, FRG) [14], intra- and inter-batch c.v. $<6 \%$. For technical reasons immunoreactive-trypsin concentrations were not available in all subjects.

TCP subjects were classified into non-diabetic (ND, $n=7$ ), impaired-glucose-tolerance (IGT, $n=7$ ) and diabetic (FCPD, $n=26$ ) according to the 1985 WHO criteria. Clinical and biochemical features of the subjects are shown in Table 1 . Of 26 diabetic subjects 16 were newly diagnosed (less than 2 weeks) while 10 had been diagnosed previously (one month to 10 years, median 1.5 years). Of the

latter, seven were treated with insulin, albeit irregularly, two with oral hypoglycaemic agents and one with diet only. Data from OGTTs on 26 normal weight, non-diabetic control subjects ( $<35$ years) without any pancreatic symptoms or a first degree family history of diabetes has been shown for comparison. These subjects (out-patients for minor illnesses and hospital staff) had volunteered in response to our appeal for control subjects. Data from 48 such subjects has been shown for immunoreactive-trypsin.

\section{Statistical analysis}

Data are presented as median and range unless specified. Nonparametric statistics (Mann Whitney U, Wilcoxon and Spearman's coefficients) are used for comparisons and correlations. Multiple regression analysis was done on data 'normalised' by logarithmic transformation whenever necessary.

\section{Results}

\section{Clinical and biochemical features (Table 1)}

Non-diabetic TCP subjects were younger than both impaired-glucose-tolerance and diabetic subjects $(p<$ $0.001)$. In keeping with this difference in age, non-diabetic

Table 1. Basic information on subjects with tropical calcific pancreatitis

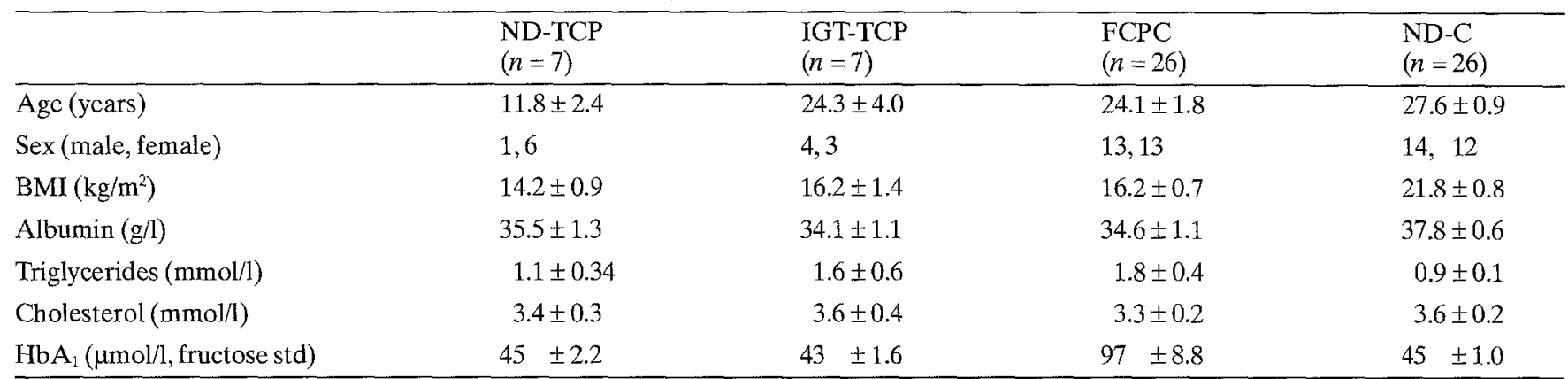

Mean \pm SEM

TCP = Tropical calcific pancreatitis; ND = Non-diabetic; IGT $=$ Impaired glucose tolerance; FCPD $=$ Fibro-calculous-pancreatic-diabetes;

ND-C = Non-diabetic control subjects 

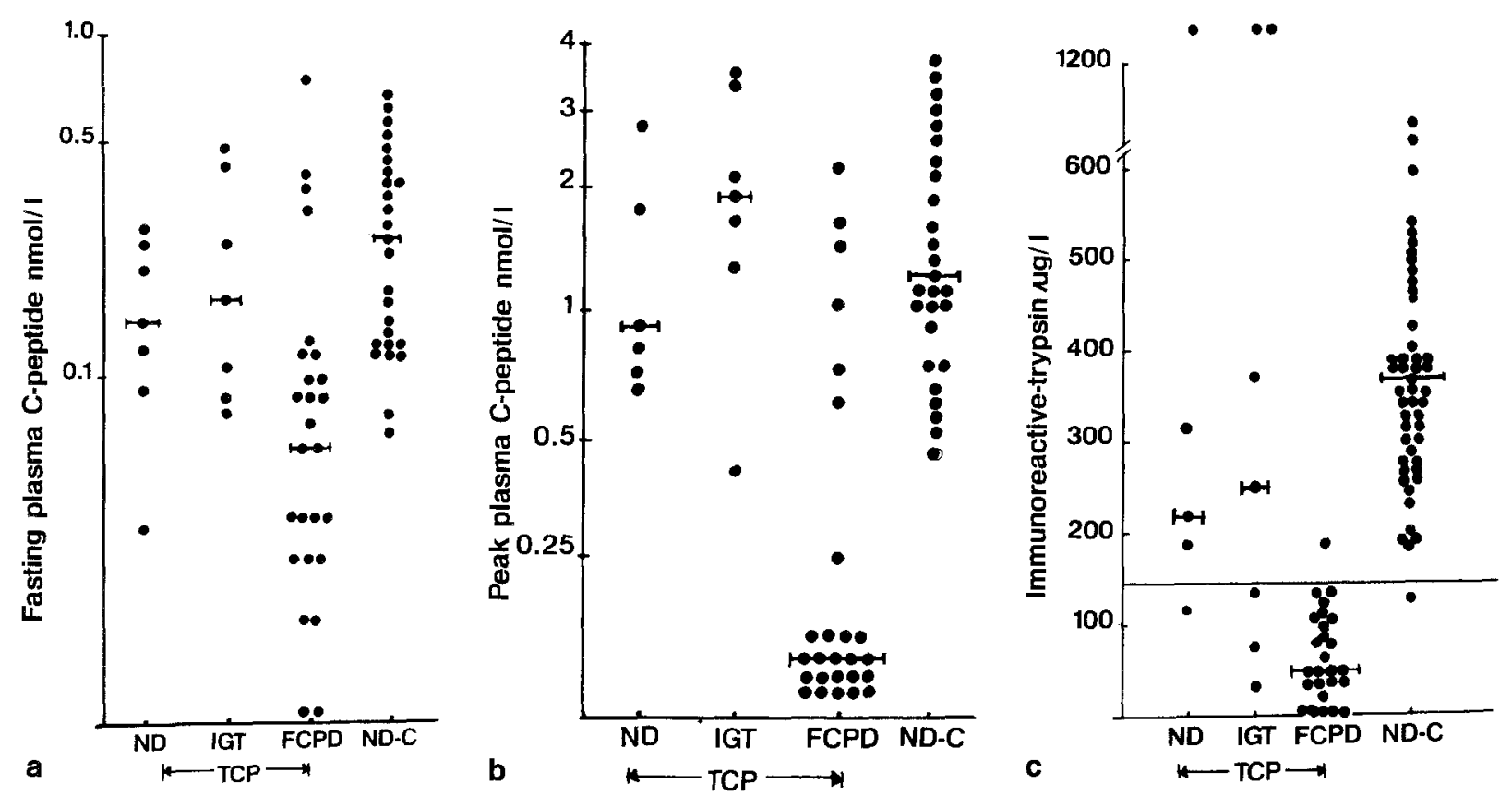

Fig. 2 a-c. Distribution of fasting plasma C-peptide (a), peak plasma C-peptide (b) and plasma immunoreactive-trypsin (c) in different groups. Abbreviations as in Figure 1. C-peptide on log scale. Medians shown by bars.

TCP subjects were shorter and lighter but there was no significant difference in BMI between the three groups. There was no difference in the age of impaired-glucosetolerance and diabetic subjects. Duration of symptoms was difficult to establish but was much shorter in nondiabetic TCP patients, the majority of whom were studied within a short time of the first severe episode of abdominal pain. Many of those with impaired-glucose-tolerance and diabetes gave a history of abdominal pain in childhood but could not provide detailed information. Plasma albumin, triglycerides and cholesterol were similar in the three groups.

Compared to the control subjects, all three groups of TCP patients had lower BMI $(p<0.01)$. Plasma albumin was lower in impaired-glucose-tolerance and diabetic subjects $(p<0.01)$ but not in non-diabetic TCP patients $(p=0.07)$, triglycerides were higher in diabetic subjects $(p<0.001)$.

\section{OGTT and Beta-cell function}

Fasting plasma glucose was elevated in those with diabetes compared to other groups $(p<0.001)$, and was higher in impaired-glucose-tolerance subjects compared to non-diabetic TCP subjects $(p<0.05)$. Plasma glucose concentrations after the glucose load are shown in Figure $1 \mathrm{a}$, and were as expected, significantly higher in both impaired-glucose-tolerance and fibro-calculous-pancreatic-diabetic subjects compared to non-diabetic TCP subjects. In the diabetic group, 14 showed fasting plasma glu- cose levels above $11 \mathrm{mmol} / \mathrm{l}$ and 12 showed $2 \mathrm{~h}$ glucose values above $22 \mathrm{mmol} / \mathrm{l}$ indicating the severity of diabetes.

Fasting plasma insulin and C-peptide concentrations (Fig. 2a) were lower in both diabetic and non-diabetic TCP than those in control subjects $(p<0.01)$, and in diabetic subjects than those in impaired-glucose-tolerance subjects $(p<0.05)$. There was a wide scatter of fasting C-peptide concentrations in diabetic subjects $(0.02$ to $0.75 \mathrm{nmol} / \mathrm{l})$ but $13(50 \%)$ were less than $0.06 \mathrm{nmol} / \mathrm{l}$. After oral glucose, plasma insulin and C-peptide concentrations rose significantly in all groups (Fig. 1b, c). The rise in diabetic subjects was, however, small and sluggish. Impaired-glucose-tolerance and non-diabetic TCP subjects showed a brisk and sustained rise which was not different from the control subjects. There was no difference between impaired-glucose-tolerance and non-diabetic TCP subjects at any time, but both showed higher concentrations than diabetic subjects at all times. Peak C-peptide levels after glucose (Fig. $2 \mathrm{~b}$ ) also showed a wide variation in diabetic subjects ( 0.06 to $2.4 \mathrm{nmol} / \mathrm{l})$ but 20 showed concentration less than $0.25 \mathrm{nmol} / 1$.

Fasting C-peptide concentrations were not related to age, sex, BMI, glucose or any of the other measured variables. Peak C-peptide concentrations were, however, inversely related to fasting $\left(r_{\mathrm{s}}=-0.54, p<0.001\right)$ as well as $2 \mathrm{~h}$ plasma glucose concentrations $\left(r_{\mathrm{s}}=-0.51, p<0.003\right)$ and $\mathrm{HbA}_{1}\left(r_{\mathrm{s}}=-0.60, p<0.001\right)$.

\section{Exocrine pancreatic marker (immunoreactive-trypsin)}

Three non-diabetic TCP subjects showed normal immunoreactive-trypsin concentration (Fig. 2c), one was subnormal and one showed a very elevated level consistent with diagnosis of pancreatitis. Two subjects with impaired-glucose-tolerance showed normal concentrations, three showed subnormal and two showed very ele- 
vated levels. Only 1 of 26 diabetic subjects showed a "normal' immunoreactive-trypsin concentration, 25 showed subnormal immunoreative-trypsin concentrations, while none showed an elevated level. Sixty six percent of diabetic subjects showed almost undetectable immunoreactive-trypsin $(<50 \mu \mathrm{g} / \mathrm{ml})$.

\section{Exocrine-endocrine correlation}

There was a direct relationship between plasma immunoreactive-trypsin and peak C-peptide concentrations $\left(r_{\mathrm{s}}=0.54, p<0.002\right)$ when all TCP subjects were considered together, as well as for those with diabetes $\left(r_{\mathrm{s}}=0.50, p<0.01\right)$ which persisted on multivariate analysis, allowing for age, BMI, fasting plasma glucose and $\mathrm{HbA}_{1}$.

\section{Discussion}

Large scale prospective epidemiologic studies of TCP are yet to be performed because of social difficulties and poor medical infrastructure in tropical countries. Most of the data available (including the present study) are from hospital based cross-sectional studies with inherent limitations, but nevertheless provide important clues.

Hyperglycaemia in chronic pancreatitis is thought to result from reduction in the Beta-cell mass below a critical level [15]. In TCP, islets are destroyed by progressive fibrosis [16] and might also be affected by mediators of inflammation liberated in the adjacent inflamed acini. In addition, morphologically normal islets may not function effectively because of the disruption of their vascular supply by the fibrotic process. It is not unreasonable, therefore, to expect that endocrine function in TCP will be diminished in parallel to declining exocrine function. This has been shown before in alcoholic pancreatitis [15]. To the best of our knowledge there are no similar studies reported in TCP.

Our results demonstrate a spectrum of exocrine pancreatic damage in TCP from non-diabetic through impaired-glucose-tolerance to diabetic state, in this cross-sectional study. Thus, elevated concentrations of the exocrine pancreatic marker (immunoreactive-trypsin) suggestive of active pancreatitis were seen in some nondiabetic TCP subjects and in some with impaired-glucosetolerance, but in none of the diabetic patients. Normal concentrations of immunoreactive-trypsin in some nondiabetic and impaired-glucose-tolerance TCP patients suggest a relatively preserved pancreatic mass. Subnormal concentrations of immunoreactive-trypsin in only one non-diabetic, $\sim 40 \%$ of impaired-glucose-tolerance and over $95 \%$ of diabetic subjects suggest progressively diminishing pancreatic exocrine reserve. Many of the fibro-calculous-pancreatic-diabetic subjects showed undetectable concentrations, demonstrating a severe pancreatic loss. Thus, pancreatitis seems to be active in the early stages but 'burnt out' in later stages. The exocrine mass seems to be relatively preserved in earlier stages but severely diminished by the time diabetes develops.
Endocrine Beta-cell function showed a similar spectrum. Thus, as a group non-diabetic and impaired-glucose-tolerance TCP subjects showed near normal plasma insulin and C-peptide concentrations while diabetic subjects showed diminished levels. Plasma C-peptide concentrations were inversely related to plasma glucose and $\mathrm{HbA}_{1}$, indicating that Beta-cell function is a major factor in glycaemic regulation in TCP. Transition to impairedglucose-tolerance and diabetes from normoglycaemia seems to occur gradually over a number of years, as seen from the ages of the three groups. Geevarghese [2] made the classic comment about TCP; "abdominal pain in childhood and diabetes in youth". Similarly, our non-diabetic TCP subjects were mostly children who were diagnosed because of the typical pain of pancreatitis, impaired-glucose-tolerance and diabetes were usually diagnosed in the second and third decades. Young age at diagnosis is a distinctive feature of TCP, in comparison to alcoholic pancreatitis which usually occurs at a much later age. It also appears that exocrine function can be considerably decreased before endocrine function is compromised, either because of a greater reserve of the latter or because the primary illness resides in the former. The diabetic group is quite heterogeneous, as previously reported [7]. Thus, six of our diabetic subjects showed residual Beta-cell function (peak plasma C-peptide $>0.25 \mathrm{nmol} / 1$ ). Twenty fibrocalculous-pancreatic-diabetic patients had very diminished Beta-cell function (Fig. 2 b), comparable to classic insulin-dependent diabetes; a majority of these were newly diagnosed but with long duration of neglected symptoms and were severely hyperglycaemic when first seen. This heterogeneity in Beta-cell (function) preservation may be multifactorial (duration, nutrition, toxic influences etc.), but the most interesting finding is the direct relationship between exocrine-endocrine parameters (immunoreactive-trypsin and C-peptide) not only for all TCP patients but even for the diabetic group alone. It supports the notion that the endocrine loss (Beta cell) in TCP is secondary to progressive exocrine loss. Alternatively, there may be a common factor, such as an environmental toxin acting simultaneously on both the components. Prospective studies of exocrine-endocrine parameters would throw more light on the natural history of TCP pancreatitis and the exocrine-endocrine relationship.

To conclude, we have demonstrated in a cross-sectional study a spectrum of exocrine-endocrine function in TCP, from the non-diabetic to the diabetic state. Progressive exocrine damage and loss seem to encroach on endocrine (Beta cell) reserve leading to hyperglycaemia. Even in the fibro-calculous-pancreatic diabetes group, there is a wide spectrum of Beta-cell function. This probably contributes to the heterogeneity in clinical-metabolic profile.

Acknowledgements. We gratefully acknowledge financial assistance of the Wellcome Trust and the British Diabetic Association, London, UK, as well as technical assistance of Ms. L. Ashworth and Ms. L. Bigham. RAS was supported by Birla Smarak Kosh, Bombay Hospital, Bombay. We are grateful to Col. V. A. Narayanan, Armed Forces Medical College, Pune for allowing us to study some of his patients. 


\section{References}

1. Balkrishnan V (1987) Tropical pancreatitis (Pancreatite tropical). In: Bernandes P, Hugier M (eds) Maladies due Pancreas Exocrine. Doin, Paris

2. Geevarghese PJ (1968) Pancreatic diabetes. Popular Prakashan, Bombay

3. McMillan DE, Geevarghese PJ (1979) Dietary cyanide and tropical malnutrition diabetes. Diabetes Care 2: 202-208

4. Diabetes Mellitus, Report of a WHO Study group (1985)WHO Tech. Rep. Ser. 727; WHO, Geneva

5. Kannan V (1981) Insulin secretion in pancreatic diabetes mellitus. J Asso Phy Ind 29: 321-331

6. Mohan V, Snehalatha C, Ramchandran A, Jayashree R, Viswanathan M (1983) Pancreatic B-cell function in tropical pancreatic diabetes. Metabolism 32: 1091-1092

7. Mohan V, Mohan R, Susheela L, Snehalatha, Bharani G, Mahajan VK, Ramchandran A, Viswanathan M, Kohner EM (1985) Tropical pancreatic diabetes in South India: heterogeneity in clinical and biochemical profile. Diabetologia 28: 229-232

8. Ahuja MMS, Sharma GP (1985) Serum C-peptide content in nutritional diabetes. Horm Metabol Res 17: 267-268

9. Vannasaeng S, Nitiyanant W, Vichayanrat A, PolybuteS, Harnthong S (1986) C-peptide secretion in calcific tropical pancreatic diabetes. Metabolism 35: 814-817

10. Samal KC, Das S, Parija CR, Tripathy BB (1987) C-peptide response to glycemic stimuli. J Asso Phy Ind 35: 362-364

11. Parker KM, England JD, Da Costa J, Hess RL, Goldstein DE (1981) Improved colorimetric assay for glycosylated haemoglobin. Clin Chem 27: 669-672

12. Morgan CR, Lazarow A (1963) Immunoassay of insulin: two antibody system. Plasma insulin levels in normal, sub-diabetic and diabetic rats. Diabetes 12: 115-126

13. Heding LG (1972) Radioimmunological determination of human C-peptide in serum. Diabetologia 11: 591-605

14. Dandona P, Elias E, Beckett AG (1978) Serum trypsin in diabetes mellitus. Br Med J 2: 1125-1126

15. Vinik AI, Jackson WPU (1980) Endocrine secretions in chronic pancreatitis. In: Podolsky S, Viswanathan M (eds) Secondary diabetes. The spectrum of the diabetic syndromes. Raven Press, New York, pp 165-189

16. Naglotimath SJ (1980) Pancreatic pathology in pancreatic calcification with diabetes. In: Podolsky S, Viswanathan M (eds) Secondary diabetes. The spectrum of the diabetic syndromes. Raven Press, New York, pp 117-145

Received: 9 October 1989

and in revised form: 12 February 1990

Dr. C.S. Yajnik

Wellcome Diabetes Study

KEM Hospital

Pune 411011

India

\section{Announcement}

\section{Lessons from Animal Diabetes 3rd International Workshop, Japan}

This workshop will take place on October 17-19 1990, at Nihon Daigaku Kaikan, 4-8-24, Kudan-minami, Chiyoda-ku, Tokyo 102, Japan. The preliminary programme consists of the following: 1 . CNS and stress on blood glucose regulation in laboratory animals; 2. Pharmacological treatment of diabetes using animals; 3 . Vasucu- lar neurological and other complications in animal diabetes; 4 . Pathogenesis of NIDDM, obesity, insulin resistance, diet and others; 5. Streptozotocin alloxian and other diabetogenic agents. Abstract deadlive: End June 1990. Further information is available from the Secretariat: Lessons From Animal Diabetes; 3rd International Workshop, Japan, Hongo SKY Building 2F, 3-38-11, Hongo, Bunkyo-ku, Tokyo 113, Japan. Tel.: 03-815-3377, Fax: 03-815-4954. 